\title{
ÍNDICE DE COBERTURA VEGETAL PELA CULTURA DO MILHO NO PERÍODO DE CHUVAS INTENSAS NO SUL DE MINAS GERAIS ${ }^{1}$
}

\author{
Plant cover index in the period of intensive rainfall for corn crop at south \\ of Minas Gerais state, Brazil
}

\author{
Fabiana Silva de Souza ${ }^{2}$, Marx Leandro Naves Silva ${ }^{3}$, Nilton Curi $^{3}$, \\ Junior Cesar Avanzi ${ }^{4}$, Renzo Garcia Von Pinho ${ }^{5}$, Gabriela Camargos Lima ${ }^{4}$
}

\begin{abstract}
RESUMO
A cobertura vegetal é a defesa natural do solo contra a erosão hídrica. Nos modelos de estimativas de perdas de solo, o efeito da cobertura vegetal na interceptação da energia cinética da chuva é a variável chave na modelagem do processo erosivo. Assim sendo, objetivou-se avaliar a eficiência da cobertura vegetal, proporcionada pela cultura do milho, e suas relações com os atributos fitotécnicos desta cultura para alguns híbridos. O estudo foi realizado no campo demonstrativo de híbridos de milho da Universidade Federal de Lavras, localizada no município de Lavras, MG. Para determinação da cobertura vegetal utilizou-se um aparato que consiste em uma estrutura horizontal, contendo orifícios para visualização dos pontos com cobertura e sem cobertura vegetal, sendo as leituras feitas de forma aleatória e transversalmente às linhas da cultura. Os atributos fitotécnicos avaliados foram altura da planta, estande, matéria seca e produção de grãos. Diante dos resultados pode-se concluir que o maior índice de cobertura vegetal foi observado para os híbridos de milho P 30F33, P 30F90, P 3021, STRIKE, FORT, VALENT, UFLA 2001, UFLA 2004, CO 32, D 8480, D 8420 DKB 333B, DKB 440, evidenciando boa qualidade como planta protetora do solo. No período de maior ocorrência de chuvas, na região sul de Minas Gerais, a cultura do milho pode minimizar o efeito do processo erosivo. A produção de matéria seca relacionou-se bem com o índice de cobertura vegetal, podendo ser um indicativo quanto à proteção do solo.
\end{abstract}

Termos para indexação: Erosão hídrica, enxurrada, conservação do solo, escoamento superficial.

\section{ABSTRACT}

The plant cover is a natural protection of soil against water erosion. In estimative models of soil loss, the effect of plant cover in the interception of rainfall kinetic energy is the key variable in the modeling of the erosive process. Thus, the aim of this work is to evaluate the efficiency of the plant cover provided by the corn crop and their relations with the phytotechnical attributes of this crop for its respective hybrids. This study was conducted in the corn hybrids demonstrative field at the Federal University of Lavras, Minas Gerais State, Brazil. In order to evaluate the plant cover a display was used that included a horizontal structure, containing holes for viewing the points with and without plant cover, the being readings performed at random and transversally to the crop lines. The phytotechnical attributes evaluated were plant height, stand, dry matter and grain production. It can be concluded that the higher plant cover index was observed for corn hybrids P 30F33, P 30F90, P 3021, STRIKE, FORT, VALENT, UFLA 2001, UFLA 2004, CO 32, D 8480, D 8420 DKB 333B, and DKB 440, proving good quality as a protective soil plant. In the period of larger occurrence of rainfall in the south of Minas Gerais state, the corn crop can minimize the effect of the erosive process. The production of dry matter was well correlated with the plant cover index, and can be used as evidence of soil protection.

Index terms: Water erosion, runoff, conservation of soil, overland flow.

(Recebido em 22 de junho de 2006 e aprovado em 17 de julho de 2009)

\section{INTRODUÇÃo}

O processo erosivo é a principal causa de degradação do solo, por ocasionar prejuízos ao setor agrícola e ao ambiente. A erosão hídrica, que resulta em efeitos mais drásticos, pois promove desagregação e o transporte das partículas do solo, acelerando o depauperamento das terras agrícolas, além de causar poluição e assoreamento de cursos de água. A perda de solo por erosão hídrica pode ser influenciada quantitativamente pela intensificação do uso e manejo do solo, sendo que a maioria das operações agrícolas utilizadas atualmente revolve e expõe superfície do solo à ação das chuvas.

A cobertura vegetal proporcionada pela cultura atua na redução dos efeitos danosos da erosão, diminuindo a força de impacto das gotas de chuva, desestruturação do

\footnotetext{
${ }^{1}$ Trabalho apresentado na XIV Reunião Brasileira de Manejo e Conservação do Solo e da Água, Cuiabá - MT. Financiado pelo CNPq. 2Universidade Federal de Viçosa/UFV - Viçosa, MG

3Universidade Federal de Lavras/UFLA - Departamento de Ciência do Solo/DCS - Cx. P. 3037 - 37200-000 - Lavras, MG - marx@ufla.br

${ }^{4}$ Universidade Federal de Lavras/UFLA - Departamento de Ciência do Solo/DCS - Lavras, MG

5Universidade Federal de Lavras/UFLA - Departamento de Agricultura/DAG - Lavras, MG
} 
solo, selamento superficial e velocidade das enxurradas, assim, qualquer mudança na cobertura vegetal afetará diretamente a taxa de escoamento superficial (Santos et al., 2000).

A cobertura vegetal é a defesa natural do solo contra a erosão hídrica. $\mathrm{O}$ efeito da vegetação pode ser assim enumerado: (a) proteção direta contra o impacto das gotas de chuva; (b) dispersão da água, interceptando-a e evaporandoa antes que atinja o solo; (c) decomposição das raízes das plantas que, formando canículos no solo, aumentam a infiltração da água; (d) melhoramento da estrutura do solo pela adição de matéria orgânica, aumentando assim sua capacidade de retenção de água; (e) diminuição da velocidade de escoamento da enxurrada pelo aumento do atrito na superfície (Bertoni \& Lombardi Neto, 1999).

Nos modelos de estimativas de perdas de solo, o efeito da cobertura vegetal na interceptação da energia cinética da chuva é variável chave na modelagem do processo erosivo. Sendo que nas áreas tropicais a erosão provocada pelo impacto das gotas de chuva é o primeiro fator responsável pelo início do processo erosivo, e quanto maior for à proporção da interceptação pelas folhas, menor será a taxa de erosão (Stocking, 1985).

Tendo em vista a importância da cobertura vegetal, sua eficiência no tocante às perdas de solo, seu índice deve ser avaliado e gerado para diversas culturas, uma vez que este é um parâmetro utilizado nos modelos de predição da erosão. Há carência em pesquisa na região do Sul de Minas Gerais que possibilitem obter informações úteis aos produtores, principalmente no que se refere à avaliação de cultivares e tratos culturais do milho (Pereira et al., 2009).

O conhecimento dos fatores envolvidos no processo erosivo apresenta grande importância para o planejamento conservacionista na atividade agrícola e suas interações ambientais.

Assim sendo, objetivou-se avaliar a eficiência da cobertura vegetal, proporcionada pela cultura do milho no decorrer do seu desenvolvimento, quantificando o índice de cobertura vegetal (IC \%) desta cultura para alguns de seus híbridos.

\section{MATERIAL E MÉTODOS}

O estudo foi realizado no campo demonstrativo de híbridos de milho do Departamento de Agricultura da Universidade Federal de Lavras, localizada no município de Lavras, MG. A altitude média da área é de 918,84 m. O clima da região é enquadrado, de acordo com a classificação de Köppen, como Cwa, com precipitação média anual de $1.529,7 \mathrm{~mm}$. A temperatura média anual é de $19,4{ }^{\circ} \mathrm{C}$ (Ometto, 1981; Brasil, 1992). O solo foi classificado de acordo com Embrapa (2006) como Latossolo Vermelho Distrófico típico.
Para determinação do índice de cobertura vegetal, utilizou-se a metodologia descrita por Stocking (1988), sendo as leituras feitas de forma aleatória e transversalmente às linhas da cultura e medidas por um aparato que consiste em uma estrutura horizontal, contendo orifícios para visualização, instalados a cerca de 1,5 $\mathrm{m}$ de altura do solo. Convencionou-se a contagem de 0 para um solo desnudo ou com restos de vegetação, de 0,5 quando a vista for parcialmente formada por vegetação e de 1,0 se for à própria vegetação. Levando-se em consideração que a cultura do milho ultrapassa a estrutura horizontal, foi colocado um dispositivo que permite a visualização de baixo para cima, conforme descrito em Stocking (1988).

Para avaliação do índice de cobertura vegetal foi utilizada a seguinte equação:

\section{IC $(\%)=\underline{\mathrm{N}^{\mathrm{o}} \text { de visões de vegetação }} \times 100$ Total de todas as visões}

Iniciaram-se as avaliações 10 dias após a semeadura e continuaram ao longo de seus estádios de desenvolvimento, estabelecidos em função da porcentagem da cobertura do solo (Wischmeier \& Smith, 1978; Maria \& Lombardi Neto, 1997). A cobertura vegetal foi avaliada em seis estádios da cultura, representando condições uniformes de recobrimento do solo e de efeitos de manejo, a saber: D - do preparo do solo ao plantio (descoberto), sendo que esse estádio ocorreu no mês de novembro de 2001 ; PL - do plantio até 35 dias depois (pós-plantio), correspondendo ao mês de dezembro de 2001; 1 - dos 35 dias aos 60 dias após o plantio (estabelecimento), correspondendo ao mês de janeiro de 2002 (Jan); 2 - dos 60 aos 80 dias depois do plantio (desenvolvimento), correspondendo ao mês de fevereiro de 2002 (Fev); 3 - dos 80 dias do plantio até a colheita (maturação), correspondendo ao início do mês de março (Mar 1); 4 - pós-colheita, correspondendo ao fím do mês de março (Mar 2). Roloff \& Bertol (1998) relataram que no estádio inicial de desenvolvimento das culturas, as estimativas do índice de cobertura vegetal devem ser mais precisas, pois, nessa fase ocorre um rápido crescimento da cultura e elevadas perdas de solo, em razão da menor proteção oferecida. Dessa maneira, o estádio PL foi enfatizado, sendo estratificado em Dez 1, Dez 2 e Dez 3, correspondendo a 10, 20 e 35 dias pós-emergência das plântulas.

$\mathrm{O}$ experimento foi conduzido no período entre novembro/2001 a março/2002, adotando um espaçamento de $0,8 \mathrm{~m}$, fez-se adubação no plantio de $500 \mathrm{~kg} \mathrm{ha}^{-1}$ do 
formulado 8:28:16 e 0,5\% de $\mathrm{Zn}$, e duas adubações em cobertura de $400 \mathrm{~kg} \mathrm{ha}^{-1}$ do formulado 20:00:20 (4 - 5 folhas) e $180 \mathrm{~kg} \mathrm{ha}^{-1}$ de uréia ( 7 - 9 folhas). Para o controle de plantas daninhas, utilizou-se em pré-emergência o produto comercial Primestra, na dosagem de $8 \mathrm{~L} \mathrm{ha}^{-1} \mathrm{e}$, para o controle de pragas, pulverizações com $250 \mathrm{~mL} \mathrm{ha}^{-1}$ de Fastac e $30 \mathrm{~mL} \mathrm{ha}^{-1}$ de Nomoute (6 folhas).

Os atributos fitotécnicos, altura da planta, estande, matéria seca e produção de grãos foram avaliados de acordo com Embrapa (1994).

O delineamento experimental utilizado foi o inteiramente casualizados, avaliando 47 híbridos em parcelas experimentais, constituídas de duas linhas de $5 \mathrm{~m}$ cada com três repetições. Os resultados de IC e produção de grãos foram submetidos à análise de variância. As comparações múltiplas de médias foram feitas pelo teste de Scott Knott a 5\%, utilizando o programa SISVAR (Ferreira, 2000). Correlações de Pearson foram estabelecidas entre o IC e os atributos fitotécnicos, matéria seca e produção de grãos, bem como a verificação da significância dos coeficientes de correlação efetuada pelo teste $\mathrm{t}$ de Student (significativo a $1 \%$ de probabilidade). Também foram ajustados modelos entre IC e os atributos citados anteriormente, estes modelos foram ajustados com interseção em zero.

\section{RESULTADOS E DISCUSSÃO}

Na Tabela 1, apresentam-se os híbridos avaliados juntamente com seus atributos fitotécnicos e índice de cobertura vegetal (IC).

Tabela 1 - Altura da planta (AP), estande, ciclo, matéria seca (MS), produção de grãos (Prod.) e índice de cobertura vegetal (IC) para os híbridos de milho avaliados.

\begin{tabular}{|c|c|c|c|c|c|c|}
\hline Híbridos & AP & Estande & Ciclo & MS & Prod. & $\mathrm{IC}$ \\
\hline & -- m --- & -- Pl ha ${ }^{-1}$--- & & \multicolumn{2}{|c|}{----- $\mathrm{kg} \mathrm{ha}^{-1}$------ } & $--\%$--- \\
\hline P 30 F33 & 2,52 & 61239 & Precoce & $\dagger$ & $13590 \mathrm{a}$ & $92,9 \mathrm{a}$ \\
\hline P $30 \mathrm{~F} 90$ & 2,83 & 59939 & Semi-precoce & $\dagger$ & $\begin{array}{l}13360 \mathrm{a} \\
11560 \mathrm{a}\end{array}$ & $93,2 \mathrm{a}$ \\
\hline P 3021 & 2,51 & 60960 & Semi-precoce & 19000 & $11560 \mathrm{~b}$ & $91,3 \mathrm{a}$ \\
\hline P 30K75 & 2,42 & 57928 & Semi-precoce & $\dagger$ & $10870 \mathrm{~b}$ & $89,4 \mathrm{~b}$ \\
\hline PL 6880 & 2,85 & 57269 & Normal & 20000 & $11000 \mathrm{~b}$ & $84,4 \mathrm{c}$ \\
\hline PL 6001 & 2,54 & 60524 & Super-precoce & $\dagger$ & $10580 \mathrm{~b}$ & $88,7 \mathrm{~b}$ \\
\hline $\begin{array}{l}\text { BRS } 3150 \\
\quad \text { XB }\end{array}$ & 2,67 & 61594 & Precoce & $\dagger$ & $10560 \mathrm{~b}$ & $89,8 \mathrm{~b}$ \\
\hline XB 7011 & 2,65 & 59276 & Precoce & $\dagger$ & $10930 \mathrm{~b}$ & $90,7 \mathrm{~b}$ \\
\hline XB 7012 & 2,55 & 59476 & Semi-precoce & $\dagger$ & $11180 \mathrm{~b}$ & $90,4 \mathrm{~b}$ \\
\hline XB 8010 & 2,51 & 59178 & Super-precoce & $\dagger$ & $10800 \mathrm{~b}$ & $90,4 \mathrm{~b}$ \\
\hline XB 8028 & 2,79 & 60493 & Semi-precoce & 18750 & $10250 \mathrm{~b}$ & $88,4 \mathrm{~b}$ \\
\hline SPEED & 2,39 & 60258 & Super-precoce & $\dagger$ & $10320 \mathrm{~b}$ & $90,1 \mathrm{~b}$ \\
\hline STRIKE & 2,78 & 59358 & Precoce Precoce & $\dagger$ & $13170 \mathrm{a}$ & 92,6 a \\
\hline FORT & 2,56 & 59458 & $\begin{array}{l}\text { Precoce } \\
\text { Precoce }\end{array}$ & 18667 & $11020 \mathrm{~b}$ & $91,6 \mathrm{a}$ \\
\hline VALENT & 2,73 & 60666 & Precoce & $\dagger$ & $11850 \mathrm{~b}$ & $93,9 \mathrm{a}$ \\
\hline UFLA 2001 & 2,61 & 59818 & Precoce & $\dagger$ & $10340 \mathrm{~b}$ & $91,4 \mathrm{a}$ \\
\hline UFLA 2004 & 2,67 & 56594 & Precoce & 20417 & $11200 \mathrm{~b}$ & $91,9 \mathrm{a}$ \\
\hline UFLA 2005 & 2,54 & 58635 & Precoce & $\dagger$ & $11810 \mathrm{~b}$ & 89,4 b \\
\hline UFLA 2002 & 2,41 & 56049 & Precoce & $\dagger$ & $9819 \mathrm{c}$ & $86,4 \mathrm{~b}$ \\
\hline
\end{tabular}


Tabela 1 - Continuação...

\begin{tabular}{|c|c|c|c|c|c|c|}
\hline D 8550 & 2,33 & 60131 & Precoce & 18750 & $10140 \mathrm{c}$ & $89,4 \mathrm{~b}$ \\
\hline CO 32 & 2,46 & 60916 & Precoce & $\dagger$ & $10150 \mathrm{~b}$ & $92,5 \mathrm{a}$ \\
\hline D 8480 & 2,30 & 59874 & Precoce & $\dagger$ & $10910 \mathrm{~b}$ & $91,4 \mathrm{a}$ \\
\hline D 8420 & 2,36 & 57761 & Precoce & $\dagger$ & $10620 \mathrm{~b}$ & $93,2 \mathrm{a}$ \\
\hline GNZ 1721 & 2,63 & 59680 & Precoce & 18562 & $10830 \mathrm{~b}$ & $90,6 \mathrm{~b}$ \\
\hline GNZ 1717 & 2,25 & 61514 & Precoce & $\dagger$ & $8834 \mathrm{c}$ & $89,8 \mathrm{~b}$ \\
\hline GNZ 3250 & 2,39 & 59973 & Super-precoce Precoce & $\dagger$ & $9317 \mathrm{c}$ & $90,7 \mathrm{~b}$ \\
\hline GNZ 3460 & 2,66 & 59255 & Precoce & $\dagger$ & $9539 \mathrm{c}$ & $88,2 \mathrm{~b}$ \\
\hline DKB 333B & 2,62 & 58274 & Semi-precoce & 23233 & $12100 \mathrm{a}$ & $91,2 \mathrm{a}$ \\
\hline DKB 215 & 2,11 & 58430 & Precoce & $\dagger$ & $9051 \mathrm{c}$ & $86,5 \mathrm{~b}$ \\
\hline DKB 350 & 2,54 & 61135 & Precoce & $\dagger$ & $11610 \mathrm{~b}$ & $89,6 \mathrm{~b}$ \\
\hline DKB 440 & 2,56 & 60780 & Precoce & $\dagger$ & $13420 \mathrm{a}$ & $94,7 \mathrm{a}$ \\
\hline AG 4051 & 2,95 & 60941 & Normal & 19725 & $13200 \mathrm{a}$ & $89,6 \mathrm{~b}$ \\
\hline AG 6690 & 2,66 & 61538 & Precoce & $\dagger$ & $11440 \mathrm{~b}$ & $89,9 \mathrm{~b}$ \\
\hline AG 9090 & 2,34 & 57981 & Precoce & $\dagger$ & $11100 \mathrm{~b}$ & $90,5 \mathrm{~b}$ \\
\hline AG 7575 & 2,66 & 61584 & Precoce & $\dagger$ & $11150 \mathrm{~b}$ & $86,6 \mathrm{~b}$ \\
\hline AGN 32A33 & 2,60 & 59489 & Normal & $\dagger$ & $9873 \mathrm{c}$ & $82,4 \mathrm{c}$ \\
\hline AGN 32A43 & 2,48 & 59491 & Precoce & $\dagger$ & $9193 \mathrm{c}$ & $83,5 \mathrm{c}$ \\
\hline AGN 2012 & 2,54 & 60935 & Precoce & 14850 & $10430 \mathrm{~b}$ & $80,0 \mathrm{~d}$ \\
\hline AGN 01104 & 2,65 & 61016 & Precoce & $\dagger$ & $9421 \mathrm{c}$ & $81,7 \mathrm{c}$ \\
\hline A 4646 & 2,79 & 58783 & Precoce & $\dagger$ & $10650 \mathrm{~b}$ & $89,7 \mathrm{~b}$ \\
\hline A 2555 & 2,52 & 58846 & Semi-precoce Precoce & $\dagger$ & $9751 \mathrm{c}$ & $86,9 \mathrm{~b}$ \\
\hline BRS 3060 & 2,78 & 59229 & Precoce & $\dagger$ & $11890 \mathrm{~b}$ & $90,4 \mathrm{~b}$ \\
\hline A 2345 & 2,51 & 60068 & Precoce & 17646 & $10840 \mathrm{~b}$ & $88,4 \mathrm{~b}$ \\
\hline SHS 5050 & 2,58 & 61708 & Super-precoce & $\dagger$ & $10520 \mathrm{~b}$ & $80,5 \mathrm{~d}$ \\
\hline SHS 5060 & 2,54 & 58761 & Precoce & $\dagger$ & $10880 \mathrm{~b}$ & $79,0 \mathrm{~d}$ \\
\hline SHS 4040 & 2,65 & 59998 & Precoce & 11740 & $9821 c$ & $78,4 \mathrm{~d}$ \\
\hline SHS 5070 & 2,38 & 60973 & Super-precoce & $\dagger$ & $10820 \mathrm{~b}$ & $83,6 \mathrm{c}$ \\
\hline $\mathrm{CV}(\%)$ & & & & & 8,22 & 2,45 \\
\hline
\end{tabular}

As médias seguidas pela mesma letra na coluna, não diferem entre si pelo teste de Scott Knott ao nível de 5\% de significância. † Não se determinou a MS $\left(\mathrm{kg} \mathrm{ha}^{-1}\right)$.

A partir dos dados apresentados na Tabela 1, observa-se o valor de IC máximo dos híbridos estudados. Os valores apresentaram uma amplitude de 78 a $95 \%$. Nesse sentido, destacam-se os híbridos P 30F33, P 30F90, P 3021, STRIKE, FORT, VALENT, UFLA 2001, UFLA 2004, CO 32, D 8480, D 8420 DKB 333B e DKB 440, proporcionando um maior valor de IC, apresentando também elevada produção de grãos (superiores a 10.000 $\mathrm{kg} \mathrm{ha-1}$ ), constituindo características desejáveis com relação à proteção do solo contra o impacto direto das gotas de água da chuva, e quanto à produção. Os demais híbridos, apesar de diferirem estatisticamente desses citados, apresentaram cobertura vegetal e produção de grãos e matéria seca satisfatória. Todos os híbridos apresentaram índice de cobertura superior a 30\% após 35 dias (estádio 1) e valores decrescentes de cobertura após 85 dias (estádio 3), em razão do ao decréscimo de área foliar da cultura, observado geralmente após esse estágio. Arruda et al. (1984) também verificaram uma grande senescência das folhas no final do ciclo produtivo da cultura.

Na Figura 1, demonstra-se a distribuição da precipitação mensal para a área de estudo no período de julho 2001 a junho de 2002. 
Na Figura 1, pode-se notar que o período de maior precipitação (novembro a março), período este que se concentra, em média, $90 \%$ da erosividade da chuva para Lavras (Silva et al., 2005; Silva et al., 2009), a cultura do milho apresenta bons índices de cobertura do solo (Figura 2), notadamente a partir de dezembro (estádio PL2). A perda de solo média, período de novembro a março, para a mesma classe de solo em parcela-padrão, foi de 14,54 $\mathrm{Mg} \mathrm{ha}^{-1}$ (Silva et al., 2005; Silva et al., 2009). Considerando um índice de proteção à ação erosiva de 30\% (híbrido DKB 333B), no mês de dezembro, essa perda de solo reduziria para valores na ordem de $10 \mathrm{Mg}$ ha $^{-1}$, que está abaixo da tolerância de perdas de solo encontrada por estes autores. Isso mostra a importância da cobertura do solo pela cultura do milho na redução das perdas de solo. Trabalho desenvolvido em Cambissolo por Bertol \& Miquelluti (1993) mostrou redução de 85\% nas perdas de solo em relação ao solo descoberto.

Na Figura 2, observa-se o comportamento do índice de cobertura vegetal no decorrer do ciclo da cultura para os híbridos DKB 333B e SHS 4040. Esses híbridos foram os que obtiveram, respectivamente, os maiores e menores valores de MS naqueles materiais em que foi avaliada, e também possuem os valores próximos aos extremos quanto à proteção do solo à ação erosiva da chuva. Mesmo o híbrido que apresentou o menor IC, nota-se que após 35 dias pósplantio (estádio 1), a cobertura proporcionada foi de 58\%, conforme discutido anteriormente.
O híbrido DKB 333B, além de apresentar um elevado índice de cobertura (Tabela 1), o mesmo possui características de interesse ao produtor, tais como, elevada produção de MS e grãos. Estas características são desejáveis tanto do ponto de vista ambiental quanto financeiro, proporcionando maiores rendimentos.

De modo geral, os valores de IC se correlacionaram com a produção de matéria seca $\left(0,78^{* *}\right)$ e a produção de grãos $(0,49 * *)$. A correlação entre IC x MS mostrou uma boa relação, pois a biomassa aérea determinada no máximo vigor vegetativo, é a responsável pela proteção do solo contra o impacto direto da gota de chuva. A correlação entre IC x Produção de grãos foi menor. Isso se deve ao fato que nem sempre o híbrido que fornece o melhor índice de cobertura necessariamente obtém a máxima produção de grãos e vice-versa.

Na Figura 3, estão os gráficos e modelos ajustados para IC em função dos atributos fitotécnicos. Pela figura pode-se verificar que a partir de uma produção de massa aérea não houve um incremento no índice de cobertura vegetal, este comportamento também foi relatado por Roloff \& Bertol (1998), para cultura do milho. O modelo entre IC e produção de grãos, não obteve um bom ajuste, isto pode ser decorrente, possivelmente, da utilização de híbridos de alto potencial produtivo, consequentemente, os índices de cobertura vegetal também foram elevados, não havendo muita diferença no IC para patamares elevados de produção.

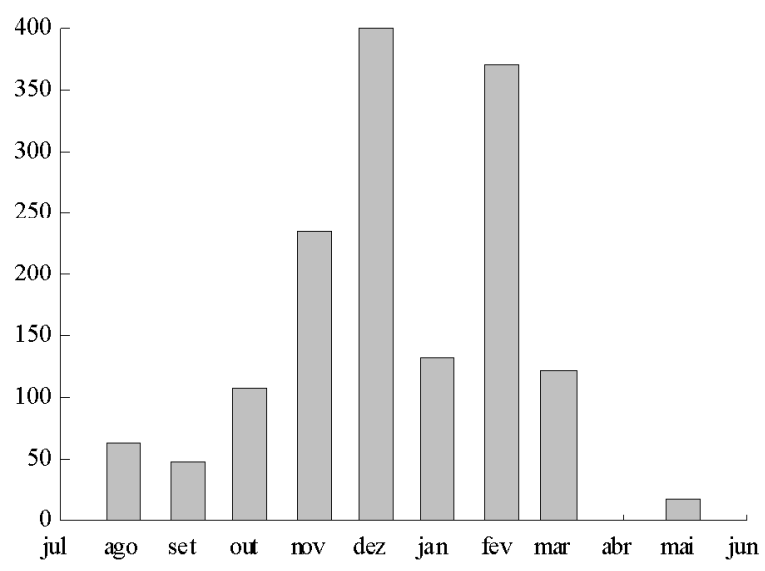

Figura 1 - Precipitação ocorrida em Lavras, no período de julho de 2001 a junho de 2002. 


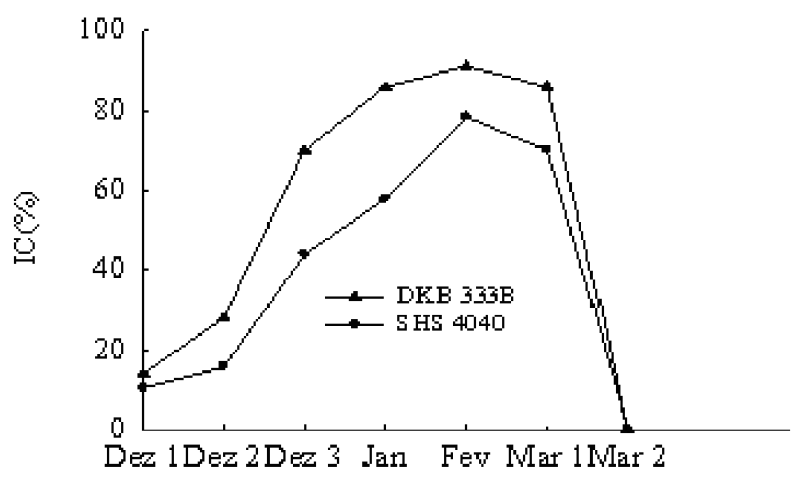

Periodo de desenvolvimento

Figura 2 - Índice da cobertura vegetal de dois híbridos de milho, de acordo com seu período de desenvolvimento, em Lavras, MG.
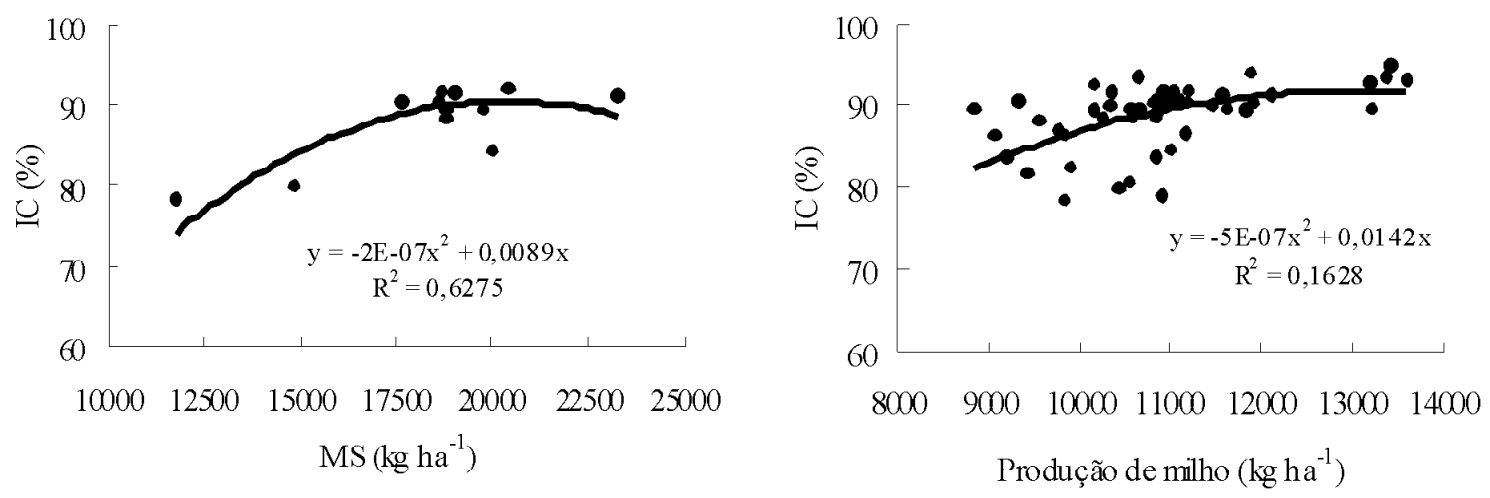

Figura 3 - Modelos ajustados para o índice de cobertura em função da produção de matéria seca de plantas e grãos.

\section{CONCLUSÕES}

O maior índice de cobertura vegetal nos híbridos de milho avaliados foi observado nos híbridos P 30F33, P 30F90, P 3021, STRIKE, FORT, VALENT, UFLA 2001, UFLA 2004, CO 32, D 8480, D 8420 DKB 333B, DKB 440, evidenciando boa qualidade como planta protetora do solo.

Dessa maneira, a utilização desses híbridos como planta protetora do solo é promissora. A cultura do milho, na região sul de Minas Gerais, pode minimizar o efeito do processo erosivo, principalmente nos períodos de maior ocorrência de chuva.

A produção de matéria seca das plantas relacionouse bem com o índice de cobertura vegetal e melhorou os atributos utilizados para estimar a proteção à erosão.

\section{REFERÊNCIAS BIBLIOGRÁFICAS}

BERTOL, I.; MIQUELLUTI, D.J. Perdas de solo, água e nutrientes reduzidas pela cultura do milho. Pesquisa
Agropecuária Brasileira, Brasília, v.28, n.10, p.12051213, 1993.

BERTONI, J.; LOMBARDI NETO, F. Conservação do solo. 4.ed. São Paulo: Ícone, 1999. 355p.

BRASIL. Ministério da Agricultura e Reforma agrária. Normais climatológicas: 1961-1990. Brasília: Secretaria Nacional de Irrigação, 1992. 84p.

EMPRESA BRASILEIRA DE PESQUISA AGROPECUÁRIA. Centro Nacional de Pesquisa de Milho e Sorgo. Relatório: ensaio nacional de milho precoce, resultado ano agrícola 1994/1995. Sete Lagoas: CNPMS, 1994. Não paginado.

EMPRESA BRASILEIRA DE PESQUISA AGROPECUÁRIA. Sistema brasileiro de classificação de solos. 2 Ed. Rio de Janeiro: Embrapa Solos, 2006. 306 p. 
FERREIRA, D.F. Análises estatísticas por meio do SISVAR - Sistema para Análise de Variância para Windows versão 4.0 In: REUNIÃO ANUAL DA REGIÃO BRASILEIRA DA SOCIEDADE INTERNACIONAL DE BIOMETRIA, 2000, São Carlos. Anais... São Carlos: UFSCar, 2000. p.255-258.

MARIA, I.C. de; LOMBARDI NETO, F. Razão de perdas de solo e fator $C$ para sistemas de manejo da cultura do milho. Revista Brasileira de Ciência do Solo, Campinas, v.21, n.2, p.263-270, 1997.

PEREIRA, J. L. A. R.; VON PINHO, R. G.; BORGES, I. D.; PEREIRA, A. M. A. R.; LIMA, T. G. Cultivares, doses de fertilizantes e densidades de semeadura no cultivo de mlho safrinha. Ciência e Agrotecnologia, v.33, n.3, p.676683, maio/jun., 2009.

OMETTO, J.C. Bioclimatologia vegetal. São Paulo: Agronômica Ceres, 1981. 440p.

ROLOFF, G.; BERTOL, O.J. Método para a estimativa da cobertura do solo e da altura do dossel de algumas culturas de verão. Revista Brasileira de Ciência do Solo, Campinas, v.22, p.319-327, 1998.

SANTOS, C.A.G.; SUZUKI, K.; WATANABE, M.; SRINIVASAN, V.S. Influência do tipo da cobertura vegetal sobre a erosão no semi-árido paraibano. Revista Brasileira de Engenharia Agrícola e Ambiental, Campina Grande, v.4, n.1, p.92-96, 2000.

SILVA, A. M.; SILVA, M. L. N.; CURI, N.; AVANZI, J. C.; FERREIRA, M. M. Erosividade da chuva e erodibilidade de Cambissolo e Latossolo na Região de Lavras, Sul de Minas Gerais. Revista Brasileira de Ciência do Solo, Viçosa, v.33, n.6, p.1811-1820, 2009.

SILVA, A.M.; SILVA, M.L.N.; CURI, N.; LIMA, J.M.; AVANZI, J.C.; FERREIRA, M.M. Perdas de solo, água, nutrientes e carbono orgânico em Cambissolo e Latossolo sob chuva natural. Pesquisa Agropecuária Brasileira, Brasília, v.40, n.12, p.1223-1230, 2005.

STOCKING, M.A. Modelagem de perdas de solo: sugestões para uma aproximação brasileira. Brasília: PNUD/FAO, 1985. 92p.

STOCKING, M.A. Assessing vegetative cover and management effects. In: LAL, R. (Ed.). Soil erosion research methods. Ankeny: Soil and Water Conservation Society, 1988. p.163-185.

WISCHEMEIER, W.H.; SMITH, D.D. Predicting rainfall erosion losses: a guide to conservation planning. Washington: USDA, 1978. 58p. (Agricultural Handbook, 537). 